\title{
Advances in the interventional management of neuropathic pain
}

\author{
Vishal Varshney $^{1,2}$, Jill Osborn $^{1,2}$, Rahul Chaturvedi ${ }^{3}$, Vrajesh Shah $^{3}$, Krishnan Chakravarthy ${ }^{4,5}$ \\ ${ }^{1}$ Department of Anesthesia, Providence Healthcare, Vancouver, BC, Canada; ${ }^{2}$ Department of Anesthesiology, Pharmacology, and Therapeutics, \\ University of British Columbia, Vancouver, BC, Canada; ${ }^{3}$ School of Medicine, University of California San Diego, La Jolla, CA, USA; ${ }^{4}$ Division of \\ Pain Medicine, Department of Anesthesiology, University of California San Diego, La Jolla, CA, USA; ${ }^{5}$ VA San Diego Health Care, San Diego, CA, \\ USA \\ Contributions: (I) Conception and design: All authors; (II) Administrative support: All authors; (III) Provision of study materials or patients: All \\ authors; (IV) Collection and assembly of data: All authors; (V) Data analysis and interpretation: All authors; (VI) Manuscript writing: All authors; (VII) \\ Final approval of manuscript: All authors. \\ Correspondence to: Krishnan Chakravarthy, MD, PhD. Department of Anesthesiology, UC San Diego Health Sciences, 3350 La Jolla Village Dr., San \\ Diego, CA 92161, USA. Email: kvchakravarthy@health.ucsd.edu.
}

\begin{abstract}
The management of neuropathic pain, defined as pain as a result of a lesion or disease in the somatosensory nervous system, continues to be researched and explored. As conventional methods demonstrate limited long-term efficacy, there is a significant need to discover therapies that offer both longitudinal and sustained management of this highly prevalent disease, which can be offered through interventional therapies. Tricyclic antidepressants (TCAs), gabapentinoids, lidocaine, serotonin norepinephrine reuptake inhibitors (SNRIs), and capsaicin have been shown to be the most efficacious pharmacologic agents for neuropathic pain relief. With respect to infusion therapies, the use of intravenous (IV) ketamine could be useful for complex regional pain syndrome, fibromyalgia, and traumatic spinal cord injury. Interventional approaches such as lumbar epidurals are a reasonable treatment choice for up to 3 months of pain relief for patients who failed to respond to conservative treatment, with a "B" strength of recommendation and moderate certainty. Neuroablative procedures like pulsed radiofrequency ablation work by delivering electrical field and heat bursts to targeted nerves or tissues without permanently damaging these structures, and have been recently explored for neuropathic pain relief. Alternatively, neuromodulation therapy is now recommended as the fourth line treatment of neuropathic pain after failed pharmacological therapy but prior to low dose opioids. Finally, the intrathecal delivery of various pharmacologic agents, such as quinoxaline-based kappa-opioid receptor agonists, can be utilized for neuropathic pain relief. In this review article, we aim to highlight advances and novel methods of interventional management of neuropathic pain.
\end{abstract}

Keywords: Neuropathic pain; interventional pain management; pharmacotherapy; spinal cord stimulation (SCS); infusion therapy

Submitted Aug 30, 2020. Accepted for publication Dec 15, 2020.

doi: $10.21037 /$ atm-20-6190

View this article at: http://dx.doi.org/10.21037/atm-20-6190

\section{Introduction}

Neuropathic pain is defined by The International Association for the Study of Pain (IASP) as pain as a result of a lesion or disease of the somatosensory nervous system (1). It can be appreciated as a syndrome of multiple lesions and diseases that result in variable signs and symptoms.
Metabolic disorders (e.g., diabetic neuropathy), viral infections [e.g., postherpetic neuralgia (PHN)], autoimmune disorders (e.g., multiple sclerosis), chemotherapy, trauma, hereditary neuropathies, inflammatory disorders and channelopathies are some of the most common pathological conditions that result in neuropathic pain (2). Neuropathic pain can alternatively be classified by the site of pathology: 
peripheral, spinal, or brain (3). Allodynia (pain due to a stimulus that does not normally provoke pain), paresthesia (an abnormal sensation, whether spontaneous or evoked), and hyperalgesia (increased pain from a stimulus that normally provokes pain) are commonly reported symptoms and signs suggestive of underlying neuropathic pain.

The prevalence of neuropathic pain has been estimated by an epidemiological systematic review to be between 3\% and $17 \%$, although it may vary due a global dissonance on the definition of neuropathic pain (4). The incidence varies by the underlying cause with a calculated 3.9-42.0/100,000 person-years, 12.6-28.9/100,000 person-years, and 15.372.3/100,000 person-years for PHN, trigeminal neuralgia and peripheral diabetic neuropathy respectively (4). Prevalence of neuropathic pain has been shown to be higher in women, those aged 50-64, and those in the occupation of manual labor.

The multitude of etiologies for neuropathic pain in addition to the variations of the primarily subjective nature of pain mandate a thorough clinical exam for the evaluation of neuropathic pain. Various questionnaires including IDPain, Douleur Neuropathique 4 (DN4), and the Leeds Assessment of Neuropathic Symptoms and Signs (LANSS) scale clinically vary in sensitivity and specificity and some lack the capability to clearly elucidate the pathologic cause for a patient's pain (5).

Currently, there are multiple pharmacologic treatments. First-line agents recommended by The Special Interest Group on Neuropathic Pain (NeuPSIG) are tricyclic antidepressants (TCAs), gabapentinoids, and selective serotoninnorepinephrine reuptake inhibitors (SNRI) (6). Tramadol, capsaicin and lidocaine can be considered as second line therapy (7). However, neuropathic pain can be difficult to treat in certain patients, requiring a combination of both pharmacologic and non-pharmacologic modalities. Clinically significant pain relief was shown to be a result of percutaneous nerve stimulation in post-amputational neuropathic pain (8). Pulsed radiofrequency for cervical radicular pain has also yielded clinically significant pain relief in a randomized control trial (9). We aim to show advances and novel methods of interventional management for neuropathic pain.

\section{Current landscape in the management of neuropathic pain}

\section{Algorithm}

The first step in establishing a comprehensive algorithm for neuropathic pain management involves an understanding of the importance of optimizing sleep, functional status, mood and the social environment of patients. Utilizing an interdisciplinary approach, involving psychologists, acupuncturists, physiotherapists, and masseuses can provide both physiologic and psychological-based reductions in pain. After establishing a multidisciplinary team, pharmacologic therapy underlies the backbone of first-line therapy for neuropathic pain. Specifically, tricyclic antidepressants (TCAs), gabapentinoids, lidocaine, SNRIs, and capsaicin are the most efficacious (10). In order to determine efficacy, physicians should select one of the above agents, such as TCAs, and trial the drug for a period of 4-6 weeks, evaluating pain relief halfway through and toward the end of that period. If ineffective, dose adjustments or an alternate medication can be utilized. Second-line therapy involves the use of combination pharmacologic agents, as studies have shown that $45 \%$ of patients with neuropathic pain requires at least two medications for adequate analgesia (11). After failure of second-line therapy, referral to a pain specialist should be considered. Pain specialists may utilize a variety of interventional measures for pain relief-such as epidural steroid injections (ESI) or pulsed radiofrequency ablation (PRFA). If these fail, a fourth-line and more invasive approach, such as neurostimulation, can be considered. Finally, low-dose opioids can be implemented and integrated as a fifth-line, albeit less optimal, treatment for refractory cases.

\section{Pharmacological review}

TCAs are thought to provide analgesic effects primarily through their inhibition of serotonin and noradrenaline reuptake, though they also block additional receptors such as histamine and sodium channels (12). Previous studies have shown that the number needed to treat (NNT) for moderate pain relief for TCAs was 3.6, with a number needed to harm (NNH) for minor adverse effects (e.g., dry mouth) of 9 (13). Duloxetine and venlafaxine, common SNRIs which block the re-uptake of serotonin and norepinephrine, have a slightly higher NNT of 6.4, with a NNH of 11.8 (14). Gabapentinoids, such as gabapentin and pregabalin, block presynaptic alpha-2-delta calcium channels in the dorsal horn of the spinal cord, thereby decreasing neurotransmitter release (10). The NNT of gabapentin has been reported to be 6.3, with a NNH of 25.6 (14). Adverse effects include sedation, dizziness and gait instability. The medications above have the advantage of less tolerance to the drug than 
alternate medications such as opioids. As an alternate to gabapentinoids, TCAs, and SNRIs, topical agents such as a lidocaine patch can be utilized, which provides analgesia by blockage of voltage-gated sodium channels in peripheral nerves. Capsaicin, an alternative to lidocaine, exerts its analgesic effects through the inhibition of transient receptor potential vanilloid sub-type 1 (TRPV1) receptors on A $\delta$ and C-nerve fibers. A Cochrane review found that the NNT for PHN and HIV-associated peripheral neuropathy for capsaicin was 10. Naltrexone, an opioid receptor antagonist, has been shown in preclinical studies to work synergistically with gabapentinoids to reduce allodynia, and has also been shown to be efficacious in treating complex regional pain syndrome (CRPS), though the data is limited $(15,16)$. Furthermore, while certain agents such as lidocaine can be applied topically, their efficacy can be improved with altered administration routes. Studies have shown that the use of subcutaneous lidocaine can decrease pain scores for patients with PHN, cancer-related pain, and second-degree burns $(17,18)$.

\section{Infusion therapies}

Ketamine, a noncompetitive antagonist of the $\mathrm{N}$-methyl$\mathrm{D}$-aspartic acid receptor, exerts its effect by modulating the transmission of ascending nociceptive information, and also modulating descending inhibitory pathways by activating pain-regulation centers of the body, such as the anterior cingulate cortex, orbital frontal cortex, insula and brainstem (19). The use of intravenous (IV) ketamine could potentially be useful for CRPS, fibromyalgia, and traumatic spinal cord injury. However, given the lack of studies on infusion dosing, duration, and rate, as well as significant adverse events, IV ketamine should not be used routinely outside of the hospital setting. For patients that are treatment resistant to standard pharmacologic agents, IV lidocaine $(5-7.5 \mathrm{mg} / \mathrm{kg})$ can be administered for pain relief, ranging from a few hours to a month, though the correlation between IV and oral administration of mexiletine is weak (20).

\section{Interventional approaches}

Current evidence points to lumbar epidurals being a reasonable treatment choice for up to 3-month relief for patients who failed to respond to conservative treatment, with a "B" strength of recommendation and moderate certainty (20). When pain originates from cervical radiculopathy and brachialgia, the use of a cervical epidural should be used only for exceptional cases and more conservative approaches should be emphasized, a recommendation with moderate certainty and a " $\mathrm{C}$ " strength of recommendation. Peripheral nerve blocks (transforaminal nerve root blocks, stellate ganglion blacks for PHN, peroneal blocks for lumbar radicular pain, etc.) can be utilized for up to 12 weeks of pain relief, though the strength of recommendations are all either grades "C" or " $D$ " (11). PRFA is a technique that delivers electrical current to the afferent nociceptive neural fibers, thereby inhibiting pain transmission. The current literature points to the use of PRFA for PHN and occipital neuralgia cases; however, the technology is less useful for trigeminal neuralgia and radicular pain (10).

\section{Neuromodulation}

Spinal cord stimulation (SCS) of the dorsal columns within the epidural space has traditionally thought to decrease pain transmission through inhibition of small nociceptive projections (A $\delta$ and $\mathrm{C}$ ) in the dorsal horn. For patients with failed back surgery syndrome and CRPS, SCS has been recommended with a "B" strength and moderate certainty. For traumatic neuropathy and brachial plexopathy, SCS use has a recommendation strength of " $\mathrm{C}$ " with moderate certainty (20). Targeted drug therapy involves delivering pharmacologic agents directly to the dorsal horn of the spinal cord, thereby decreasing first pass metabolism and increasing potency. For non-radicular pain, current American Pain Society (APS) guidelines state that there is insufficient evidence for utilizing intrathecal therapy for opioids or any alternate drugs, though conclusive evidence for neuropathic and radicular pain has not been determined. The current consensus according to recent studies indicates that target therapy can be considered for patients requiring $>50$ morphine equivalent dose (MED) of opioids, with a strong recommendation for its use if requirements exceed 90 MED (10).

\section{Recent advances in interventional management of neuropathic pain}

\section{Infusion therapies}

As described above, infusion therapies are suggested in the treatment algorithm for neuropathic pain. While lidocaine and ketamine continue to be arguably the two 
most common IV medications used for neuropathic pain treatment, other medications continue to be studied as our understanding of neuropathic pain pathophysiology improves.

Oxytocin, a neuropeptide produced in the supraoptic and paraventricular nuclei of the hypothalamus, has shown effects in pre-synaptic inhibition of $\mathrm{A} \delta$ and $\mathrm{C}$-fiber signals at nociceptive-specific and wide dynamic range neurons in the dorsal horn of the spinal cord (21). This mechanism of pain modulation at the dorsal horn is thought to be a predominant hypothesis for its management of neuropathic pain, and is why animal studies have sought to validate the use of oxytocin for both nociceptive and neuropathic pain. An animal study from 2005 assessed electrical and mechanical pain tolerance in 12 Sprague-Dawley rats with induced neuropathic pain (22). They applied varying dosages of oxytocin and discovered that oxytocin increased pain tolerance in neuropathic but not normal rats. Despite the relatively robust findings from animal research with regards to IV oxytocin therapy, there is a paucity of human subject research on its effects in chronic pain conditions, and especially in neuropathic pain.

In CRPS, IV bisphosphonates have been established as a potential therapy in refractory cases. A randomized, doubleblind, placebo-controlled study comparing IV neridronate to placebo looked at 82 patients with CRPS type 1 (23). Patients were given $100 \mathrm{mg}$ IV neridronate over 2 hours, for 4 treatments over 10 days. In the 40 days following infusion, visual analog scale remained unchanged in the placebo group and further decreased in the active group by $46.5 \mathrm{~mm}$ (95\% CI: $-52.5,-40.5)$ vs. $22.6 \mathrm{~mm}$ (95\% CI: $-28.8,-16.3)$ for placebo group $(\mathrm{P}<0.0001)$, on a 100 -point Visual Analogue Scale (VAS) scale. Their results were so encouraging that they concluded "bisphosphonates should be considered the first choice of treatment for CRPS type 1". No serious side effects have been noted with IV bisphosphonate therapy in meta-analyses observing clinical efficacy (24).

\section{Neuroablative procedures}

Neuroablative procedures like PRFA work by delivering electrical field and heat bursts to targeted nerves or tissues without permanently damaging these structures (25). Novel approaches in how these electrical field and/or heat bursts are delivered to nerves have sought to reduce adverse effects like sensory loss, dysesthesia, and rebound neuralgia, while simultaneously improving longevity of pain relief and symptoms.
Recent studies have shown the potential utility of low-temperature plasma radiofrequency ablation (coblation) technology as a treatment modality for neuropathic pain (26). This technology utilizes radiofrequency energy to excite the electrolytes that are present in a conductive medium such as saline, and thereby forms energized plasma. The discharge plasma then creates radical species that then interact with organic tissue and have sufficient energy to split molecular bonds, leading to tissue dissolution at low temperatures of $40-70^{\circ} \mathrm{C}$. Coblation may suppress dorsal root ganglion (DRG) stimulation, and thereby reduces the erroneous ectopic input to the central nervous system-the potential mechanism for neuropathic pain relief.

Cryoneurolysis works by freezing nerves, preventing conduction of nerve impulses to the sensory system. Despite being clinically in use since 1976, it has been underutilized as a modality in neuropathic pain. Systems allowing ultrasound-guided cryoneurolysis have been recently developed to allow broader, more widespread use of this modality for refractory neuropathic pain. A recent prospective study evaluated 22 patients with refractory peripheral neuropathy who received percutaneous cryoablation, and pain scores were recorded at 1, 3, 6, 9 and 12 months post-procedure (27). Mean pain levels on a 10 -point visual analog scale were $8.3 \pm 1.9$ before intervention and $2.3 \pm 2.5$ at 1 month, $3.2 \pm 2.5$ at 3 months, $4.7 \pm 2.7$ at 6 months, and $5.1 \pm 3.7$ at 12 months afterward. These were statistically significant decreases between pre- and post-procedural pain scores. There were no complications reported from the procedures.

\section{Neuromodulation}

Since Dr. Shealy first pioneered neuromodulation via SCS as an implantable therapy in 1967 , its clinical effectiveness and success in treating neuropathic pain has advanced considerably (28). Neuromodulation is now recommended as the fourth line treatment of neuropathic pain after failed pharmacological therapy but before low dose opioids (10).

Management of neuropathic pain is the most common reason for using neuromodulation. As a therapy, this can be further broken down into SCS, DRG stimulation and peripheral nerve stimulation (PNS). In addition, with the improvement in technology and increasing understanding of neuromodulation, different modes of stimulation have further improved its efficacy and indications for treatment. The Neuromodulation Appropriateness Consensus Committee (NACC) of the International Neuromodulation 
Society (INS) published consensus papers for SCS in 2014 and for DRG stimulation in 2018, with a review of the evidence to date and recommendations of when each therapy is appropriate $(29,30)$. A recent systematic review demonstrated that SCS leads to a decrease in pain intensity when compared with placebo therapy (31). Indications for neuromodulation have expanded to include axial back pain, peripheral neuropathy (including human immunodeficiency virus neuropathy) and diabetic neuropathy, abdominal/ pelvic pain, postamputation pain, and postsurgical pain syndromes including post-thoracotomy, post-inguinal hernia repair, and post knee surgery pain (29).

The mechanism for neuromodulation's therapeutic response is not fully understood but it appears to be associated with peripheral (distal to the DRG), spinal cord/ segmental (DRG and spinal cord) and supraspinal effects depending upon the condition, the location of stimulation, and the mode of stimulation (32). Conventional tonic stimulation $(40-100 \mathrm{~Hz})$ effects were originally explained by the Gate Control Theory where activation of the largediameter afferents (A-beta fibers) resulted in inhibition of small (A $\delta$ and C) fibers (33). However, all the effects of SCS could not be explained by this theory. Research has shown that neuromodulation results in local changes of wide dynamic neuron excitability, facilitation of physiologic inhibition mechanisms, and changes in activation of neurotransmitters including gamma aminobutyric acid (GABA), glutamate, adenosine, acetylcholine, substance $\mathrm{P}$, calcitonin gene-related peptide (CGRP), brain-derived neurotrophic factor (BDNF), and bradykinins (33). Supraspinal mechanisms for the effects of SCS have also been shown to alter electroencephalogram potentials and cerebral blood flow in functional magnetic resonance imaging (fMRI) studies $(34,35)$.

With the advancement of technology, new leads and modes of stimulation beyond tonic stimulation have further improved the efficacy of neuromodulation in neuropathic pain. DRG stimulation has allowed for more focused treatment and improved outcomes in conditions that were challenging to treat. Initial studies with DRG stimulation and lower limb CRPS suggested improved pain reduction and vasomotor changes (36). Further discrete areas of pain are more easily targeted with DRG stimulation such as post inguinal hernia surgery pain. High Frequency stimulation $(10 \mathrm{kHz})$ has shown to be effective in relieving pain, reducing disability, and improving quality of life, specifically in patients with axial low back and leg pain (37). Differential target multiplexed (DTM) stimulation is another mode of stimulation that appears promising in the treatment of low back pain (38). DTM uses multiple electrical signals varying in frequency, pulse width and amplitude. Larger randomized studies are required to determine its efficacy. Non-linear burst stimulation utilizes $40 \mathrm{~Hz}$ bursts with five spikes at $500 \mathrm{~Hz}$ per burst at a rate of 40 bursts per second to modulate neuronal activity in the medial and lateral pathways of the brain (thought to conduct affective components of pain), and has been shown to provide better pain relief than conventional tonic stimulation (39-41).

Neuromodulation is now on the cutting edge of treatment as pharmacological interventions have repeatedly failed with significant side effects and risks of addiction. It appears then that neuromodulation may be the future of neuropathic pain management. Multicenter, randomized controlled trials, and research of mechanisms must be further done to demonstrate its therapeutic indications and cost effectiveness.

\section{Intrathecal drug delivery}

Several physicians noted decreased research and development surrounding intrathecal analgesic medications during the 2019 North American Neuromodulation Society meeting, as ziconotide continued to be the only US Food and Drug Administration (FDA) approved drug for intrathecal delivery since 2003. Alternate pharmacologic agents, such as corticosteroids, gabapentin and ketamine have either demonstrated decreased efficacy or unacceptable adverse events, such as spinal cord toxicity when delivered intrathecally (42).

Quinoxaline-based kappa-opioid receptor agonists, which have a better safety profile, have thereby acquired increasing attention (43). These agonists exert a stronger analgesic effect by targeting the kappa-opioid receptor, while circumventing the unwanted effects of mu-opioid receptors such as euphoria, dependence and respiratory depression (44). The preclinical data show that the intrathecal delivery of kappa-opioid receptor agonists leads to anti-inflammatory activity-downregulating the proliferation, activation and secretion of cytokines in the immune system (43). After a neural insult, inflammatory mediators are released by nearby immune cells in the spinal cord, which thereby activate nociceptive neurons in the area and induce pain (45). Intrathecal administration of Kappa-opioid receptor agonists may help decrease the inflammatory process and the activation of these nociceptive neurons. 
The mechanism of chronic neuropathic pain, though not clearly understood, may be related to changes in anionic gradients that are associated with microglial cells (46). Recent studies show that glial cells, along with other immune cells, may lead to the development of chronic pain by increasing neuroinflammation, cross-talking with other neural cells, and secreting pain molecules (47). One potential therapy that targets glial cells which previously has been validated for neurodegenerative disorders, antisense oligonucleotides (ASOs) administration intrathecally, is currently being evaluated for chronic neuropathic pain (48). Preclinical studies utilizing animal models have shown that injecting ASOs intrathecally can inhibit microglia and thereby decreases mechanical allodynia following peripheral nerve injury. This process may occur by decreasing the gene expression of protein receptors that are involved in microglial activation and thereby in the pathogenesis of chronic neuropathic pain (49).

Interestingly botulinum toxin, which has been validated previously for intramuscular use for migraine relief, has also demonstrated efficacy for the treatment of neuropathic pain via the intramuscular or intraarticular routes (50). Botulinum may damage spinal substance $\mathrm{P}$ receptors, thereby inhibiting the downstream cascade involved in pain conduction to the brain. Though there are some studies that discuss spinal toxicity related to the intrathecal administration of botulinum, new synthetic botulinum conjugates have been created that are not toxic and are fast-acting (51). Preclinical data utilizing mouse models conducted by Maiarù $e t$ al. showed that single intrathecal administration of synthetic botulinum conjugates led to prolonged pain relief in neuropathic pain models, and lacked any clinically significant adverse effects (52).

The TRPV1 receptor, which is primarily located in both the peripheral and central terminals of sensory neurons, has been shown to be an important receptor in the conductance and alteration of neuropathic pain signals (53).

After nerve insults, the inflammatory mediators that are released sensitize and upregulate the expression of TRPV1 receptors, which thereby induces chronic allodynia and hyperalgesia (54). Though it would be reasonable to assume that antagonism of these receptors would provide relief, inhibition paradoxically leads to elevation in pain scores (55). Agonism of the TRPV1 receptors, therefore, has been shown to reduce hyperalgesia, most likely by leading to constant depolarization and thereby sustained inactivation of the TRPV1 receptor (56). Resiniferatoxin (RTX), a strong agonist of the TRPV1 receptor, has demonstrated high selectivity for the TRPV1 receptor when administered intrathecally, and has fortunately not shown any significant side effects when administered (57). Preclinical data explored the effect of intrathecal RTX for allodynia reduction in mouse models, and found that RTX is efficacious in reducing allodynia (58)-however, this finding and safety profiles have not been reproduced or demonstrated in human clinical trials.

\section{Applications for the future}

As novel methods in the interventional management of neuropathic pain continue to be elucidated, the future may lie in two domains: interventions with multimodal mechanisms, and interventions that offer longevity in therapy without tolerance. Non-ablative implantable therapies, such as neuromodulation, demonstrate promise in these domains. Interventional management of neuropathic pain extends beyond cessation of nerve signal transmission, and therapies that target not just signal transmission, but signal modulation and processing (i.e., non-linear burst stimulation, closed-loop stimulation) can also lead to longevity of therapy. Certainly, as neuropathic pain continues to be a burden, the need to investigate and develop these novel methods becomes even more important.

\section{Acknowledgments}

Funding: None.

\section{Footnote}

Provenance and Peer Review: This article was commissioned by the Guest Editor (Dr. Antonio Ruggiero) for the series "Pain Therapy" published in Annals of Translational Medicine. The article has undergone external peer review.

Conflicts of Interest: All authors have completed the ICMJE uniform disclosure form (available at http://dx.doi. org/10.21037/atm-20-6190). The series "Pain Therapy" was commissioned by the editorial office without any funding or sponsorship. Dr. KC reports other from Abbott, Bioness, Medtronic, Nalu Medical, Saluda Medical, and Newrom Biomedical, outside the submitted work. The other authors have no other conflicts of interest to declare.

Ethical Statement: The authors are accountable for all aspects of the work in ensuring that questions related 
to the accuracy or integrity of any part of the work are appropriately investigated and resolved.

Open Access Statement: This is an Open Access article distributed in accordance with the Creative Commons Attribution-NonCommercial-NoDerivs 4.0 International License (CC BY-NC-ND 4.0), which permits the noncommercial replication and distribution of the article with the strict proviso that no changes or edits are made and the original work is properly cited (including links to both the formal publication through the relevant DOI and the license). See: https://creativecommons.org/licenses/by-nc-nd/4.0/.

\section{References}

1. IASP Terminology - IASP [Internet]. [cited 2020 Aug 17]. Available online: https://www.iasp-pain.org/Education/ Content.aspx? ItemNumber $=1698$

2. Colloca L, Ludman T, Bouhassira D, et al. Neuropathic pain. Nat Rev Dis Prim 2017;3:17002.

3. Gilron I, Baron R, Jensen T. Neuropathic pain: principles of diagnosis and treatment. Mayo Clin Proc 2015;90:532-45.

4. van Hecke O, Austin SK, Khan RA, et al. Neuropathic pain in the general population: a systematic review of epidemiological studies. Pain 2014;155:654-62. Erratum in: Pain. 2014 Sep;155(9):1907.

5. Mathieson S, Maher CG, Terwee CB, et al. Neuropathic pain screening questionnaires have limited measurement properties. A systematic review. J Clin Epidemiol 2015;68:957-66.

6. Dworkin $\mathrm{RH}, \mathrm{O}^{\prime}$ Connor $\mathrm{AB}, \mathrm{Kent} \mathrm{J}$, et al. Interventional management of neuropathic pain: NeuPSIG recommendations. Pain 2013;154:2249-61.

7. Cavalli E, Mammana S, Nicoletti F, et al. The neuropathic pain: An overview of the current treatment and future therapeutic approaches. Int J Immunopathol Pharmacol 2019;33:2058738419838383.

8. Gilmore C, Ilfeld B, Rosenow J, et al. Percutaneous peripheral nerve stimulation for the treatment of chronic neuropathic postamputation pain: A multicenter, randomized, placebo-controlled trial. Reg Anesth Pain Med 2019;44:637-45.

9. Van Zundert J, Patijn J, Kessels A, et al. Pulsed radiofrequency adjacent to the cervical dorsal root ganglion in chronic cervical radicular pain: A double blind sham controlled randomized clinical trial. Pain 2007;127:173-82.

10. Bates D, Schultheis BC, Hanes MC, et al. A
Comprehensive Algorithm for Management of

Neuropathic Pain. Pain Med 2019;20:S2-S12.

11. Tarride JE, Collet JP, Choinière $M$, et al. The economic burden of neuropathic pain in Canada. J Med Econ 2006;9;55-68.

12. Obata H. Analgesic Mechanisms of Antidepressants for Neuropathic Pain. Int J Mol Sci 2017;18:2483.

13. Saarto T, Wiffen PJ. Antidepressants for neuropathic pain. Cochrane Database of Systematic Reviews 2007. doi: 10.1002/14651858.CD005454.pub2.

14. Finnerup NB, Attal N, Haroutounian S, et al. Pharmacotherapy for neuropathic pain in adults: A systematic review and meta-analysis. Lancet Neurol 2015;14:162-73.

15. Pineda-Farias JB, Caram-Salas NL, Salinas-Abarca $\mathrm{AB}$, et al. Ultra-Low Doses of Naltrexone Enhance the Antiallodynic Effect of Pregabalin or Gabapentin in Neuropathic Rats. Drug Dev Res 2017;78:371-80.

16. Chopra P, Cooper MS. Treatment of complex regional pain syndrome (CRPS) using low dose naltrexone (LDN). J Neuroimmune Pharmacol 2013;8:470-6.

17. Edwards AD. The role of systemic lidocaine in neuropathic pain management. J Intraven Nurs 1999;22:273.

18. Ni J, Wang X, Tang Y, et al. Subcutaneous Injection of Triamcinolone and Lidocaine to Prevent Postherpetic Neuralgia. Pain Physician 2017;20:397-403.

19. Maher DP, Chen L, Mao J. Intravenous Ketamine Infusions for Neuropathic Pain Management. Anesth Analg 2017;124:661-74.

20. Mailis A, Taenzer P. Evidence-based guideline for neuropathic pain interventional treatments: Spinal cord stimulation, intravenous infusions, epidural injections and nerve blocks. Pain Res Manag 2012;17:150-8.

21. Rash JA, Aguirre-Camacho A, Campbell TS. Oxytocin and Pain: A Systematic Review and Synthesis of Findings. Clin J Pain 2014;30:453-62.

22. Condés-Lara M, Maie I, Dickenson A. Oxytocin actions on afferent evoked spinal cord neuronal activities in neuropathic but not in normal rats. Brain Res 2005; 1045:124-33.

23. Varenna M, Adami S, Rossini M, et al. Treatment of complex regional pain syndrome type I with neridronate: a randomized, double-blind, placebo-controlled study. Rheumatology 2013;52:534-42.

24. Chevreau M, Romand X, Gaudin P, et al. Bisphosphonates for treatment of Complex Regional Pain Syndrome type 1: A systematic literature review and meta-analysis of randomized controlled trials versus placebo. Joint Bone 
Spine 2017;84:393-9.

25. Chang MC. Efficacy of Pulsed Radiofrequency Stimulation in Patients with Peripheral Neuropathic Pain: A Narrative Review. Pain Physician 2018;21:E225-34.

26. Li H, Li Y, Guo Z, et al. Low-temperature plasma radiofrequency ablation in phantom limb pain: A case report. Brain Circ 2018;4:62-4.

27. Yoon JHE, Grechushkin V, Chaudhry A, et al. Cryoneurolysis in Patients with Refractory Chronic Peripheral Neuropathic Pain. J Vasc Interv Radiol 2016;27:239-43

28. Shealy CN, Mortimer JT, Reswick JB. Electrical inhibition of pain by stimulation of the dorsal columns: preliminary clinical report. Anesth Analg 1967;46:489-91.

29. Deer TR, Mekhail N, Provenzano D, et al. The appropriate use of neurostimulation of the spinal cord and peripheral nervous system for the treatment of chronic pain and ischemic diseases: The neuromodulation appropriateness consensus committee. Neuromodulation 2014;17:515-50.

30. Deer TR, Pope JE, Lamer TJ, et al. The Neuromodulation Appropriateness Consensus Committee on Best Practices for Dorsal Root Ganglion Stimulation. Neuromodulation 2019;22:1-35.

31. Duarte R V., Nevitt S, McNicol E, et al. Systematic review and meta-analysis of placebo/sham controlled randomised trials of spinal cord stimulation for neuropathic pain. Pain 2020;161:24-35.

32. Jensen MP, Brownstone RM. Mechanisms of spinal cord stimulation for the treatment of pain: Still in the dark after 50 years. Eur J Pain 2019;23:652-9.

33. Sdrulla AD, Guan Y, Raja SN. Spinal Cord Stimulation: Clinical Efficacy and Potential Mechanisms. Pain Pract 2018;18:1048-67.

34. Bocci T, De Carolis G, Paroli M, et al. Neurophysiological Comparison Among Tonic, High Frequency, and Burst Spinal Cord Stimulation: Novel Insights Into Spinal and Brain Mechanisms of Action. Neuromodulation 2018;21:480-8.

35. Stancák A, Kozák J, Vrba I, et al. Functional magnetic resonance imaging of cerebral activation during spinal cord stimulation in failed back surgery syndrome patients. Eur J Pain 2008;12:137-48.

36. Deer TR, Levy RM, Kramer J, et al. Dorsal root ganglion stimulation yielded higher treatment success rate for complex regional pain syndrome and causalgia at 3 and 12 months: A randomized comparative trial. Pain 2017;158:669-81.
37. Kapural L, Yu C, Doust MW, et al. Novel 10-kHz High-frequency Therapy (HF10 Therapy) Is Superior to Traditional Low-frequency Spinal Cord Stimulation for the Treatment of Chronic Back and Leg Pain. Anesthesiology 2015;123:851-60.

38. Fishman MA, Calodney A, Kim P, et al. Prospective, Multicenter Feasibility Study to Evaluate Differential Target Multiplexed Spinal Cord Stimulation Programming in Subjects With Chronic Intractable Back Pain With or Without Leg Pain. Pain Pract 2020;20:761-8.

39. Chakravarthy K, Fishman MA, Zuidema X, et al. Mechanism of Action in Burst Spinal Cord Stimulation: Review and Recent Advances. Pain Med 2019;20:S13-22.

40. Chakravarthy K, Kent AR, Raza A, et al. Burst Spinal Cord Stimulation: Review of Preclinical Studies and Comments on Clinical Outcomes. Neuromodulation 2018;21:431-9.

41. Chakravarthy K, Malayil R, Kirketeig T, et al. Burst Spinal Cord Stimulation: A Systematic Review and Pooled Analysis of Real-World Evidence and Outcomes Data. Pain Med 2019;20:S47-57.

42. Jamison DE, Cohen SP, Rosenow J. Chapter 76 Implanted Drug Delivery Systems for Control of Chronic Pain. Essentials of Pain Medicine (Fourth Edition). Elsevier, 2018:693-702.e2.

43. Tangherlini G, Kalinin DV, Schepmann D, et al. Development of Novel Quinoxaline-Based $\kappa$-Opioid Receptor Agonists for the Treatment of Neuroinflammation. J Med Chem 2019;62:893-907.

44. Stein C, Lang LJ. Peripheral mechanisms of opioid analgesia. Curr Opin Pharmacol 2009;9:3-8.

45. Costigan M, Scholz J, Woolf C. Neuropathic pain: a maladaptive response of the nervous system to damage. Annu Rev Neurosci 2009;32:1-32.

46. Coull JA, Beggs S, Boudreau D, et al. BDNF from microglia causes the shift in neuronal anion gradient underlying neuropathic pain. Nature 2005;438:1017-21.

47. Grace PM, Hutchinson MR, Maier SF, et al. Pathological pain and the neuroimmune interface. Nat Rev Immunol 2014;14:217-31.

48. Luo X, Fitzsimmons B, Mohan A, et al. Intrathecal administration of antisense oligonucleotide against p38 $\alpha$ but not p38 $\beta$ MAP kinase isoform reduces neuropathic and postoperative pain and TLR4-induced pain in male mice. Brain Behav Immun 2018;72:34-44.

49. Chen G, Luo X, Qadri M, et al. Sex-Dependent Glial Signaling in Pathological Pain: Distinct Roles of Spinal Microglia and Astrocytes. Neurosci Bull 2018;34:98-108. 50. Park J, Park HJ. Botulinum Toxin for the Treatment of 
Neuropathic Pain. Toxins (Basel) 2017;9:260.

51. Brown DC, Agnello K. Intrathecal substance P-saporin in the dog: efficacy in bone cancer pain. Anesthesiology 2013;119:1178-85.

52. Maiarù $M$, Leese $C$, Certo $M$, et al. Selective neuronal silencing using synthetic botulinum molecules alleviates chronic pain in mice. Sci Transl Med 2018;10:eaar7384.

53. Latorre R, Brauchi S, Orta G, et al. ThermoTRP channels as modular proteins with allosteric gating. Cell Calcium 2007;42:427-38.

54. Kissin I, Davison N, Bradley EL Jr. Perineural resiniferatoxin prevents hyperalgesia in a rat model of postoperative pain. Anesth Analg 2005;100:774-80, table of contents.

Cite this article as: Varshney V, Osborn J, Chaturvedi R, Shah $\mathrm{V}$, Chakravarthy K. Advances in the interventional management of neuropathic pain. Ann Transl Med 2021;9(2):187. doi: 10.21037/atm-20-6190
55. Haanpää M, Treede R. Capsaicin for neuropathic pain: Linking traditional medicine and molecular biology. Eur Neurol 2012;68:264-75.

56. Szallasi A, Blumberg PM. Resiniferatoxin and its analogs provide novel insights into the pharmacology of the vanilloid (capsaicin) receptor. Life Sci 1990;47:1399-408.

57. Iadarola M, Mannes A. The vanilloid agonist resiniferatoxin for interventional- based pain control. Top Med Chem 2011;2171-9.

58. Parisi JR, Laura A, Andrade M De, et al. Antiallodynic effect of intrathecal resiniferatoxin on neuropathic pain model of chronic constriction injury. Acta Neurobiol Exp (Wars) 2017;77:317-22. 